\title{
ORAL TYROSINE TOLERANCE TEST IN RHEUMATOID ARTHRITIS
}

\author{
BY \\ J. L. KALLIOMÄKI, A. LEHTONEN, AND P. SEPPÄLÄ \\ From the Department of Medicine, University of Turku, Finland
}

On the basis of their observations that the patients with collagen diseases, but not their controls, excrete 2.5 dihydroxy phenylpyruvic acid in the urine, and that the urinary and plasma tyrosine levels of these patients are increased, Nishimura and his associates consider that the collagen diseases are disorders of the tyrosine metabolism (Nishimura, Yasui, Okamoto, Kanazawa, Kotake, and Shibata, 1958; Nishimura, Maeda, Yasui, Okamoto, Matsunaka, and Toshina, 1961; Nishimura, 1963).

Rivlin, Melmon, and Sjoerdsma (1965) have introduced an oral tyrosine tolerance test, using a fluorometric method for estimation of tyrosine in the plasma. Adopting this test, we have endeavoured to study the metabolism of tyrosine in rheumatoid arthritis because the question is still open for discussion.

\section{Material and Methods}

Our series consists of fifteen patients with rheumatoid arthritis ( 4 men, 11 women; average age $48 \cdot 6$ years), and sixteen control subjects with various internal diseases ( 4 men, 12 women; average age 53.7 years). Some clinical characteristics of the patients with rheumatoid arthritis are shown in Table $I$ and the diagnoses of the controls in Table II. All the patients of the two groups were treated in hospital during the study. No drugs were given on the day before the test or on the test day.

The tyrosine tolerance test was performed as suggested by Rivlin and others (1965). We used 1-tyrosine puriss. (Fluka AG Buchs SG, Switzerland) and the dose given was $50 \mathrm{mg} . / \mathrm{kg}$. orally in orange juice. The drug was administered in the morning, $10 \mathrm{ml}$. of blood first being drawn for estimation of the 0 -value of the blood tyrosine level. The same amount of blood was drawn at $\frac{1}{2}$, $1 \frac{1}{2}, 3,4 \frac{1}{2}$, and 6 hours after ingestion of the test dose of tyrosine. Heparin was added as an anticoagulant. Duplicate plasma samples were run through the entire procedure. The Aminco-Bowman spectrophotofluorometer was used for fluorescence measurement, and the fluorometric method presented by Waalkes and Udenfriend (1957) for estimation of tyrosine in plasma.

\section{Results}

These are given in Tables I and II (overleaf).

The plasma tyrosine level is significantly $(t=$ 3.02; $\mathrm{P}<0.01$ ) lower in the rheumatoid arthritis group than in the control group. The differences in the plasma tyrosine level in the two groups after oral ingestion of tyrosine are statistically insignificant. In the rheumatoid arthritis group there were six patients with a plasma tyrosine level $<11 \cdot 0 \mu \mathrm{g}$./ $\mathrm{ml}$. The mean increase in the plasma tyrosine level after the test dose did not deviate significantly in these cases as compared with those with an initial tyrosine level $\geqslant 11.0 \mu \mathrm{g} . / \mathrm{ml}$. If the changes are expressed as a percentage of the control value, thedifference between the two groups is still insignificant.

The patients with rheumatoid arthritis noticed no changes in their joint symptoms during and after the tyrosine tolerance test.

\section{Discussion}

In our patients with rheumatoid arthritis the plasma level was significantly lower than in the controls. This observation is not new. Waalkes and Udenfriend (1957) reported seven patients with rheumatoid arthritis whose plasma tyrosine level seemed to be lower than that of the healthy adults included in their series. Nettelbladt and Sandell (1963), using paper chromatography, studied the content of seventeen amino acids in the serum of patients with rheumatoid arthritis, and recorded low values for arginine, glutamine, tyrosine, and histidine, while the concentration of glutamic acid was higher in patients with rheumatoid arthritis than in healthy subjects. Kulonen and Kulonen (1960) studied the blood and urinary amino acids in rheumatoid arthritis also by paper chromatography. No difference specific to rheumatoid arthritis was observed in the blood, but the urinary elimination of glycine, glutamine, and alanine was increased, and the basic amino acids (cysteine, glutamic acid, and threonine) 
TABLE I

RESULTS OF TYROSINE LOADING TEST IN 15 PATIENTS WITH RHEUMATOID ARTHRITIS

\begin{tabular}{|c|c|c|c|c|c|c|c|c|c|c|c|c|c|c|}
\hline \multirow{2}{*}{$\begin{array}{l}\text { Case } \\
\text { No. }\end{array}$} & \multirow[t]{2}{*}{ Sex } & \multirow{2}{*}{$\begin{array}{l}\text { Age } \\
\text { (yrs) }\end{array}$} & \multirow{2}{*}{$\begin{array}{c}\text { Dura- } \\
\text { tion of } \\
\text { Disease } \\
\text { (yrs) }\end{array}$} & \multirow{2}{*}{$\begin{array}{c}\text { Stage } \\
\text { of } \\
\text { Disease }\end{array}$} & \multirow{2}{*}{$\begin{array}{c}\text { Waaler- } \\
\text { Rose } \\
\text { Titre }\end{array}$} & \multirow{2}{*}{$\begin{array}{l}\text { Latex } \\
\text { Test }\end{array}$} & \multirow{2}{*}{$\underset{(\mathbf{m m} . / \mathbf{h r})}{\text { E.S.R. }}$} & \multirow{2}{*}{$\underset{(\mathbf{g} / 100 \mathrm{ml} .)}{\mathrm{Hb}}$} & \multicolumn{6}{|c|}{ Tyrosine Loading ( $\mu \mathrm{g} . / \mathrm{ml}$.) (hrs) } \\
\hline & & & & & & & & & $\mathbf{0}$ & $\frac{1}{2}$ & $1 \frac{1}{\mathbf{z}}$ & 3 & $4 \frac{1}{2}$ & 6⿳亠丷厂甲 \\
\hline $\begin{array}{r}1 \\
2 \\
3 \\
4 \\
5 \\
6 \\
7 \\
8 \\
9 \\
10 \\
11 \\
12 \\
13 \\
14 \\
15\end{array}$ & $\begin{array}{l}\text { Female } \\
\text { Female } \\
\text { Male } \\
\text { Male } \\
\text { Female } \\
\text { Female } \\
\text { Female } \\
\text { Female } \\
\text { Female } \\
\text { Female } \\
\text { Female } \\
\text { Female } \\
\text { Female } \\
\text { Male } \\
\text { Male }\end{array}$ & $\begin{array}{l}53 \\
31 \\
32 \\
26 \\
58 \\
62 \\
51 \\
37 \\
52 \\
\mathbf{6 5} \\
\mathbf{5 3} \\
\mathbf{4 7} \\
\mathbf{6 5} \\
\mathbf{4 7} \\
\mathbf{5 1}\end{array}$ & $\begin{array}{r}1 \\
1 \\
5 \\
1 \\
\frac{1}{5} \\
40 \\
10 \\
2 \\
2 \\
2 \\
15 \\
10 \\
20 \\
1 / 12 \\
11\end{array}$ & $\begin{array}{r}\text { I I } \\
\text { III } \\
\text { III } \\
\text { III } \\
\text { III } \\
\text { III } \\
\text { II } \\
\text { IV } \\
\text { III } \\
\text { III } \\
\text { III } \\
\text { II } \\
\text { II }\end{array}$ & $\begin{array}{c}128 \\
0 \\
0 \\
0 \\
\text { lacking } \\
32 \\
8 \\
16 \\
8 \\
\text { lacking } \\
32 \\
256 \\
\text { lacking } \\
0 \\
8\end{array}$ & $\begin{array}{c}3+ \\
0 \\
0 \\
0 \\
2+ \\
3+ \\
2+ \\
3+ \\
2+ \\
\text { lacking } \\
3+ \\
3+ \\
\text { lacking } \\
2+ \\
2+\end{array}$ & $\begin{array}{r}136 \\
60 \\
60 \\
22 \\
25 \\
65 \\
53 \\
83 \\
75 \\
115 \\
113 \\
73 \\
56 \\
37 \\
71\end{array}$ & $\begin{array}{r}10 \cdot 5 \\
9 \cdot 8 \\
12 \cdot 1 \\
11 \cdot 4 \\
13 \cdot 0 \\
11 \cdot 8 \\
12 \cdot 7 \\
9 \cdot 9 \\
12 \cdot 5 \\
10 \cdot 0 \\
9 \cdot 6 \\
10 \cdot 6 \\
13 \cdot 8 \\
10 \cdot 2 \\
13 \cdot 6\end{array}$ & $\begin{array}{r}12 \cdot 0 \\
10 \cdot 5 \\
9.8 \\
9.7 \\
13 \cdot 8 \\
12.0 \\
15 \cdot 3 \\
9 \cdot 7 \\
12.8 \\
12.5 \\
15 \cdot 3 \\
8.5 \\
14.5 \\
12.8 \\
10.0\end{array}$ & $\begin{array}{l}21 \cdot 5 \\
13 \cdot 3 \\
17 \cdot 0 \\
21 \cdot 0 \\
16 \cdot 3 \\
18 \cdot 2 \\
18 \cdot 7 \\
23 \cdot 1 \\
17 \cdot 3 \\
15 \cdot 3 \\
45 \cdot 3 \\
21 \cdot 8 \\
24 \cdot 0 \\
15 \cdot 0 \\
15 \cdot 0\end{array}$ & $\begin{array}{l}29 \cdot 5 \\
20 \cdot 5 \\
29 \cdot 6 \\
27 \cdot 5 \\
28 \cdot 8 \\
17 \cdot 8 \\
27 \cdot 8 \\
23 \cdot 8 \\
17 \cdot 3 \\
16 \cdot 8 \\
48 \cdot 5 \\
22 \cdot 5 \\
24 \cdot 5 \\
20 \cdot 5 \\
21 \cdot 5\end{array}$ & $\begin{array}{l}33 \cdot 0 \\
18 \cdot 8 \\
28 \cdot 5 \\
30 \cdot 3 \\
22 \cdot 8 \\
24 \cdot 5 \\
28 \cdot 0 \\
23 \cdot 8 \\
21 \cdot 5 \\
19 \cdot 3 \\
35 \cdot 0 \\
21 \cdot 8 \\
33 \cdot 3 \\
17 \cdot 6 \\
20 \cdot 5\end{array}$ & $\begin{array}{c}24 \cdot 5 \\
14 \cdot 1 \\
23 \cdot 0 \\
21 \cdot 8 \\
25 \cdot 8 \\
29 \cdot 8 \\
28 \cdot 0 \\
23 \cdot 7 \\
25 \cdot 8 \\
18 \cdot 1 \\
24 \cdot 5 \\
\text { lacking } \\
29 \cdot 8 \\
17 \cdot 3 \\
19 \cdot 0\end{array}$ & 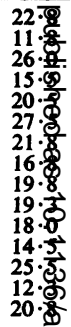 \\
\hline \multicolumn{9}{|c|}{ Mean Increase of Blood Tyrosine Content from Control Value } & & $8 \cdot 2$ & $13 \cdot 1$ & $13 \cdot 3$ & $10 \cdot 1$ & $7 \cdot 8$ \\
\hline \multicolumn{9}{|c|}{ Mean Plasma Tyrosine Content } & $\begin{array}{c}11 \cdot 9 \\
0.60 \\
2 \cdot 32\end{array}$ & $\begin{array}{c}20 \cdot 2 \\
2 \cdot 00 \\
7 \cdot 65\end{array}$ & $\begin{array}{c}25 \cdot 1 \\
2 \cdot 02 \\
7 \cdot 85\end{array}$ & $\begin{array}{r}25 \cdot 2 \\
1 \cdot 50 \\
5 \cdot 82\end{array}$ & $\begin{array}{l}23 \cdot 2 \\
1 \cdot 28 \\
4 \cdot 80\end{array}$ & $\begin{array}{r}19.91 \\
1.03 \\
4.86 \\
06\end{array}$ \\
\hline
\end{tabular}

TABLE II

RESULTS OF TYROSINE LOADING TEST IN 16 CONTROL SUBJECTS

\begin{tabular}{|c|c|c|c|c|c|c|c|c|c|}
\hline \multirow{2}{*}{$\begin{array}{l}\text { Case } \\
\text { No: }\end{array}$} & \multirow{2}{*}{ Sex } & \multirow{2}{*}{$\begin{array}{r}\text { Age } \\
\text { (yrs) }\end{array}$} & \multirow{2}{*}{ Diagnosis } & \multicolumn{6}{|c|}{ Tyrosine Loading ( $\mu \mathrm{g} . / \mathrm{ml}$ ) (hrs) } \\
\hline & & & & $\mathbf{0}$ & $\frac{1}{2}$ & $1 \frac{1}{2}$ & 3 & $4 \frac{1}{2}$ & 6 \\
\hline $\begin{array}{r}1 \\
2 \\
3 \\
4 \\
5 \\
6 \\
7 \\
8 \\
9 \\
10 \\
11 \\
12 \\
13 \\
14 \\
15 \\
16\end{array}$ & $\begin{array}{l}\text { Female } \\
\text { Male } \\
\text { Female } \\
\text { Male } \\
\text { Female } \\
\text { Female } \\
\text { Female } \\
\text { Female } \\
\text { Female } \\
\text { Female } \\
\text { Male } \\
\text { Female } \\
\text { Female } \\
\text { Female } \\
\text { Female } \\
\text { Male }\end{array}$ & $\begin{array}{l}44 \\
62 \\
48 \\
58 \\
59 \\
40 \\
43 \\
61 \\
51 \\
55 \\
39 \\
67 \\
70 \\
50 \\
72 \\
40\end{array}$ & $\begin{array}{l}\text { Observation } \\
\text { Cardiac infarction } \\
\text { Abdom. pains } \\
\text { Hemiparesis } \\
\text { Hypertensio art. } \\
\text { Migraena } \\
\text { Peptic ulcer } \\
\text { Hypertensio art. } \\
\text { Little stroke } \\
\text { Hypertensio art. } \\
\text { Haematuria } \\
\text { Little stroke } \\
\text { Angina pectoris } \\
\text { Cardiac infarction } \\
\text { Little stroke } \\
\text { Observation }\end{array}$ & $\begin{array}{l}12 \cdot 0 \\
16 \cdot 3 \\
13 \cdot 8 \\
11 \cdot 8 \\
11 \cdot 8 \\
10 \cdot 8 \\
15 \cdot 0 \\
13 \cdot 0 \\
17 \cdot 6 \\
16 \cdot 3 \\
13 \cdot 2 \\
16 \cdot 0 \\
16 \cdot 0 \\
14 \cdot 8 \\
19 \cdot 8 \\
13 \cdot 3\end{array}$ & $\begin{array}{l}22 \cdot 3 \\
16 \cdot 8 \\
19 \cdot 4 \\
16 \cdot 3 \\
19 \cdot 3 \\
18 \cdot 0 \\
25 \cdot 5 \\
16 \cdot 3 \\
31 \cdot 8 \\
33 \cdot 3 \\
25 \cdot 6 \\
24 \cdot 8 \\
22 \cdot 8 \\
27 \cdot 8 \\
24 \cdot 0 \\
20 \cdot 0\end{array}$ & $\begin{array}{l}23 \cdot 3 \\
28 \cdot 0 \\
19 \cdot 5 \\
24 \cdot 5 \\
22 \cdot 3 \\
27 \cdot 8 \\
31 \cdot 3 \\
17 \cdot 5 \\
42 \cdot 0 \\
50 \cdot 3 \\
34 \cdot 0 \\
37 \cdot 5 \\
22 \cdot 8 \\
23 \cdot 8 \\
31 \cdot 8 \\
20 \cdot 5\end{array}$ & $\begin{array}{l}25 \cdot 8 \\
23 \cdot 3 \\
19 \cdot 8 \\
25 \cdot 5 \\
20 \cdot 5 \\
31 \cdot 8 \\
38 \cdot 8 \\
19 \cdot 3 \\
31 \cdot 0 \\
38 \cdot 3 \\
19 \cdot 0 \\
27 \cdot 0 \\
30 \cdot 0 \\
35 \cdot 5 \\
41 \cdot 8 \\
24 \cdot 0\end{array}$ & $\begin{array}{c}27 \cdot 8 \\
22 \cdot 8 \\
21 \cdot 3 \\
24 \cdot 0 \\
\text { lacking } \\
21 \cdot 5 \\
30 \cdot 0 \\
\text { lacking } \\
27 \cdot 3 \\
32 \cdot 8 \\
17 \cdot 6 \\
25 \cdot 8 \\
23 \cdot 0 \\
28 \cdot 8 \\
27 \cdot 9 \\
23 \cdot 0\end{array}$ & 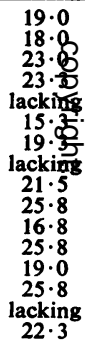 \\
\hline \multicolumn{4}{|c|}{$\begin{array}{l}\text { Mean Increase of Blood Tyrosine Content from } \\
\text { Control Value }\end{array}$} & & $8 \cdot 3$ & $14 \cdot 1$ & $13 \cdot 7$ & $10 \cdot 6$ & $6 \cdot 5$ \\
\hline \multicolumn{4}{|c|}{$\begin{array}{ll}\text { Mean Plasma Tyrosine Content } & \\
\text { SEM } \\
\text { SD }\end{array}$} & $\begin{array}{r}14 \cdot 5 \\
0 \cdot 62 \\
2 \cdot 48\end{array}$ & $\begin{array}{l}22 \cdot 8 \\
1 \cdot 29 \\
5 \cdot 15\end{array}$ & $\begin{array}{l}28 \cdot 5 \\
2 \cdot 25 \\
9 \cdot 00\end{array}$ & $\begin{array}{l}28 \cdot 2 \\
1 \cdot 86 \\
7 \cdot 45\end{array}$ & $\begin{array}{l}25 \cdot 3 \\
1 \cdot 05 \\
3 \cdot 94\end{array}$ & $\begin{array}{r}21 \cdot 1 \\
0 \cdot 94 \\
3 \cdot 39\end{array}$ \\
\hline
\end{tabular}

decreased in patients with rheumatoid arthritis compared with cases of infection and with healthy controls. The elimination of tyrosine, phenylalanine, and leucines was increased in both rheumatoid arthritis and in cases of infection.

Rivlin and others (1965) observed very high plasma tyrosine levels after oral tyrosine loading in hyperthyroidism and low values in hypothyroidism. It has been suggested that thyroidectomy may aggravate rheumatoid arthritis or that this condition may develop after such an operation (Laine, Vainio, and Holopainen, 1954; Kalliomäki, 1954; Kalliola, Kalliomäki, and Rintala, 1957).
The results here presented show that, although the plasma tyrosine level is initially low in rheumatoie arthritis, its increase and decrease after oral tyrosine loading is normal. From earlier studies we know that the urinary elimination of tyrosine is increased in rheumatoid arthritis. This discrepancy-lo basal level in plasma, normal "clearance" from plas $\mathrm{ma}$, and increased urinary elimination-is difficuits to explain. The tyrosine content of the tissues and the renal handling of tyrosine during this kind of loading test must be evaluated more closely. Thic increased extracellular fluid phase observed in rheumatoid arthritis (Kalliomäki, Kirpilä, Koskine@̣ 
and Laine, 1958) must also be taken into consideration in this connexion.

\section{Summary}

(1) An oral tyrosine tolerance test $-50 \mathrm{mg} . / \mathrm{kg}$. 1-tyrosine being given orally in the morning and the plasma tyrosine level followed thereafter by a fluorometric method for 6 hours-was performed on fifteen patients with rheumatoid arthritis and on sixteen patients with various internal disorders.

(2) The initial plasma tyrosine level was significantly $(P<0.01)$ lower in the rheumatoid patients than in the controls.

(3) The change in plasma tyrosine content after oral tyrosine loading was, within the limits of statistical error, the same in both groups of patients.

\section{REFERENCES}

Kalliola, H., Kalliomäki, J. L., and Rintala, A. (1957). Ann. med. intern. Fenn., 46, 97.

Kalliomäki, J. L. (1954). Ann. rheum. Dis., 13, 341.

—, Kirpilä, J., Koskinen, H.-M., and Laine, V. A. I. (1958). Acta rheum. scand., 4, 79.

Kulonen, M., and Kulonen, E. (1960). Scand. J. clin. Lab. Invest., 12, 84.

Laine, V. A. I., Vainio, K. J., and Holopainen, T. E. (1954). Ann. rheum. Dis., 13, 250.

Nettelbladt, E., and Sandell, B. M. (1963). Ibid., 22, 269.

Nishimura, N. (1963). J. chron. Dis., 16, 343.

-, Maeda, K., Yasui, M., Okamoto, H., Matsunaka, M., and Toshina, H. (1961). A.M.A. Arch. Derm., 83, 644.

—, Yasui, M., Okamoto, H., Kanazawa, M., Kotake, Y., and Shibata, Y. (1958). Ibid., 77, 255.
Rivlin, R. S., Melmon, K. L., and Sjoerdsma, A. (1965). New Engl. J. Med., 272, 1143.

Waalkes, T. P., and Udenfriend, S. (1957). J. Lab. clin. Med., 50, 733.

Le test de tolérance à la tyrosine par voie buccale dans l'arthrite rhumatismale

RÉSUMÉ

(1) Le test de tolérance à la tyrosine par voie buccale$50 \mathrm{mg} . / \mathrm{kg}$. de 1-tyrosine le matin, suivi pendant 6 heures de détermination fluorométrique du taux plasmatique de la tyrosine - fut effectué chez 15 malades atteints d'arthrite rhumatismale et 16 malades atteints de diverses affections internes.

(2) Le taux plasmatique initial de la tyrosine fut significativement $(P<0.01)$ plus bas chez les malades rhumatisants que chez les témoins.

(3) L'élévation du contenu du plasma en tyrosine après la surcharge par voie buccale fut, dans les limites de l'erreur statistique, le même dans les deux groupes de malades.

El test de tolerancia a la tirosina por vía oral en la artritis reumatoide

\section{SUMARIO}

(1) Se efectuó un test de tolerancia a la tirosina por vía oral-50 mg. $/ \mathrm{kg}$. de 1-tirosina por la mañana, seguidos de la determinación fluorométrica por seis horas de las cifras plasmáticas de tirosina-en 15 enfermos con artritis reumatoide y 16 enfermos con varios trastornos internos.

(2) Las cifras plasmáticas iniciales de tirosina fueron significativamente $(P<0.01)$ más bajas en los enfermos reumáticos que en los demás.

(3) El cambio en la concentración plasmática de la tirosina después de su sobrecarga por vía oral fué, en los límites del error estadístico, igual en ambos grupos de enfermos. 